\title{
AM I BORING YOU? A SOCIAL STUDY TO MEASURE THE PERCEPTION OF USE OF ADOPTION AND PERCEPTIONS OF SMARTWATCH TECHNOLOGY
}

\author{
Justin Fruehauf, Robert Morris University, fruehaufj@rmu.edu \\ Fahad Al-Khalifa, Robert Morris University,fak5757@gmail.com
}

\begin{abstract}
The purpose of this study is to understand the social perceptions of smartwatch technology adoption. Specifically, it examined undergraduate and graduate Information Systems students' perceptions of the use of smartwatch technology through elements of the Unified Theory for the Acceptance and Use of Technology (Venkatesh et al., pp. 446, 2003). While the results of the study did not cover a large enough population to be generalizable, they do demonstrate interesting themes suggesting different perceptions between males and females regarding the social use of smartwatch technologies. Furthermore, the results indicate a disconnect between people's perceptions of their own actions with smartwatch technology as compared to perceptions of others' use of the technology in social situations.
\end{abstract}

Keywords: Smartwatch, Adoption, Acceptance, Unified Theory for Acceptance and Use of Technology

\section{INTRODUCTION}

Smartwatch technology represents a growing technology phenomenon due to its close ties to smart phone development. JP Morgan defines "smartwatches" as "wrist-worn wearable devices, which come under the category of 'Lifestyle Computing (Infotainment)'. They promise an enhanced user experience, acting as an extension to the smartphone, as well as a customized app-eco-system that adds on to its functionality (fitness trackers, games, etc.)" (Park, 2014). Furthermore, JP Morgan predicts "the smartwatch market size could reach US\$26 billion, assuming 180 million+ units by 2018" (Park, 2014). Powered by operating system (OS) software from Google and Apple, these devices are rapidly becoming status symbols. This parallels the use of traditional wristwatches in the same manner (Pearson et al., 2014). This sizable economic impact represents a trend towards greater user acceptance and use of smartwatch devices.

Previous studies measured the acceptance of smartwatch technology from the usage perspective of the user. However, there is a lack of research when it comes to the smartwatch and its impact on social interactions and cultural situations. Culture and traditions play a role in accepting this technology. The Unified Theory of Acceptance and Use of Technology (UTAUT) will be the base of the study to measure people's attitudes toward the smartwatch and it usage in social situations (Venkatesh, Morris, Davis, \& Davis, 2003). Using UTAUT will expose the social acceptance of the technology from both the user and those engaged in interactions with the user.

\section{LITERATURE REVIEW}

As of 2016 little has been written regarding the social perceptions stemming from the introduction of smartwatch technologies. A review of the literature shows that much of what has been researched pertains to market acceptance of smartwatch technologies and what smartwatch users seek in the technology as a sales indicator. Even in this context, however, there are key indicators of changing social behaviors due to smartwatch technology use. One study suggests that smartwatch manufacturers believed the ease of checking messages, or "glanceability", would be an advantage of smartwatch technology marketing to consumers (Giang et al., 2015). Yet the study's results showed that participants engaged the smartwatch technology faster than smart phones in order to check for new messages, but took longer to use smartwatch technology to "glance" through the message. Furthermore, "glancing" took longer than with smart phones and necessitated the use of two hands to scroll through a message compared to one 


\section{Issues in Information Systems}

Volume 17, Issue III, pp. 199-208, 2016

hand with smart phones (Giang et al., 2015). The researchers conclude that this change in behavior for checking messages may have a large impact on vehicle control by smartwatch users (Giang et al., 2015). This conclusion demonstrates a change in user interaction due to the adoption of smartwatch technology.

Chen and Shih utilized the "Unified Theory of Acceptance and Use of Technology" (UTAUT) as a model for determining the market acceptance of smartwatches based upon the usability of the watch (Chen and Shih, 2014). Chen and Shih define this usability as an ability "to assess whether consumers are willing to use the new product equipped wearable technology and the probability of actual buying behavior" (Chen and Shih, 2014, p. 4). While such an analysis is useful for understanding the motivations of those who own or want to purchase a smartwatch, it gives little insight into the social perceptions and attitudes towards the use of smartwatch technology.

The work of Pearson, Robinson, and Jones focuses on the use of smartwatches as a display device not only for the user wearing the watch, but also for actors interacting with the device owner (Pearson et al., 2014). While their study examines user and actor interactions regarding smartwatches, it focuses on the social acceptability of the actor viewing the user's watch - in particular, whether this act is an invasion of privacy or socially acceptable (Pearson et al., 2014). The researchers are not concerned with the social and cultural implications of this action, but rather use it as a platform for examining the possibility of using smartwatch technology as a means of conducting public media displays (Pearson et al., 2014).

As noted above, the focus of smartwatch technology literature has been to study methods of increasing the usage and acceptance of the technology. According to Yang, et al., "little research has been done on user acceptance and behaviors concerning them because they are still in the very early stage of commercialization" (Yang et al., 2015). In order to examine the potential social and cultural barriers associated with use of smartwatches, in particular with regards to actor attitudes about users' use of the technology during interactions, one must look to studies conducted about smart phone adoptions.

A 2011 study by Oulasvirta, Rattenbury and company addressed such social interactions through the examination of a "checking habit" (2011). Through their studies the researchers found that smart phone users formed an addiction to checking their phones. The habit would begin as an occasional action and become progressively more frequent. Over time, according to the study, this habit encroached on face-to-face interactions with others, thus interfering with the user's lifestyle (Oulasvirta et al., 2011).

Despite the lack of literature regarding smartwatch technology and social perceptions of its use, much can be gleaned from a review of how smartwatches' parent technology, smart phones, have evolved in a social frame. A study conducted by Wang, Xiang, and Fesenmaier reported on the use of smart phone technology as an instrument of change for perceptions and attitudes in travelers (Wang et al., 2016). As part of the study, the researchers collected data from participants about their attitudes toward smart phone adoption. Participants stated that using smart phones were changing them in many aspects of "everyday living" (Wang et al., 2016). Among other things, the users reported that smart phones changed the way they used downtime (Wang et al., 2016).

\section{On-Line Media}

While a dearth of information regarding the social impact of smartwatch technology exists, an online search of the issue using Internet search engines such as Google.com gives a different outcome. Although these articles may not represent formal studies on the topic, they are relevant nonetheless. On-line discussion of the topic demonstrates the concern and recognition of the possibility of changing behaviors from smartwatch technology users. An understanding of the views expressed by those in on-line media is relevant to framing any study in the area of social attitudes of smartwatch technology usage.

A 2015 CNN.com article by Andre Spicer discusses the potential social impact of smartwatch behavior (Spicer, 2015). Citing previous data that suggests that smart phone users check their devices over 150 times per day, Spicer projects the possibility of smartwatch users experiencing a "phantom effect" of checking for messages even when not wearing the watch. Citing the ability of using smartwatches to check not only messages, but also track user biodata, Spicer concludes that smartwatch technology adoption will result in users becoming self-obsessed (Spicer, 2015). 
A USAToday.com article by Steven Petrow (2015) provides etiquette tips for using smartwatches in public. Petrow presents six behavioral statements intended to be a "primer" (Petrow, 2015). Included in the list are two demonstrating the concern for behavioral changes in users of smartwatch technology:

- "Avoid raising your arm: Yes, the Activate on Wrist feature is a cool way to enable the watch's display - as a response to a 'tap notification' - but hardly subtle. It's even more rude than checking your watch in front of someone" (Petrow, 2015).

- "Don't watch your smart watch when driving" (Petrow, 2015)

Again the subject of driving is mentioned in a news media outlet. While the primary focus of this is due to safety concerns when driving, it underscores a behavioral shift due to the use of smartwatch technology. This shift is yet another form of social changes. The social implications of smartwatch use are clearly addressed with the statement regarding "raising the arm" (Petrow, 2015). Petrow's referring to this as "rude" (2015) demonstrates a distinct social perception regarding the behavior.

Specific to smartwatch technology, website www.smartwatches.org offers sections to those interested in using smartwatches. Site contributor Briley Kenney authored a January 2015 article entitled "'Smart' Device, Smartwatch and Wearable Etiquette is Evolving" profiling smartwatch acceptance issues. Key among her points is the proper conduct for smartwatch use. Of particular note, the author cites the banning of smartwatch technology in classrooms and testing centers of United States universities and colleges such as Weber State (Kenney, 2015). While student cheating is not the focus of this study, this action by school administrators exhibits social acceptance conflict with regard to the technology. What is more intriguing is that while smartwatch usage and behavior is the focal point of her commentary, at no point does Kenney write about the behavioral pattern of checking a smartwatch while interacting with others.

As has been stated numerous times, there is little literature regarding the social behaviors regarding smartwatch users and interactions with others. Conversely, a survey of the topic using Internet media produces numerous results from mainstream news outlets as well as the smartwatch user community. This awareness of the issue by the population at large combined with the lack of published research demonstrates the need for a more thorough examination of smartwatch technology and its social implications. Based upon these results, the researchers derived the following research question: How is the act of habitually checking messages and alerts on a smartwatch perceived by others engaged in a face-to-face interaction with the smartwatch-wearer?

\section{Theoretical Basis}

The study relies on the Unified Theory of Acceptance and Use of Technology (UTAUT) first described by Venkatesh and colleagues in 2003 (Venkatesh et al., 2003). In particular, the areas of "Social Influence" and "Attitude (Venkatesh et al., 2003, p. 460) of the undergraduate and graduate students are examined. Based upon the findings from their 2003 study (that led to the development of the UTAUT), Venkatesh and colleagues perceive the "Social Influence" factor is only critical in environments in which a technology is mandatory. Furthermore, they suggest that age and gender play a further role in social perception of a technology (Venkatesh et al., 2003, p. 469). Conversely, the researchers do not believe that the users' "attitude factor" for a technology is "a significant influence on behavioral intention" (Venkatesh et al., 2003, p. 456). This study uses the UTAUT factors as a means of understanding and assessing the attitudes and perceptions of smartwatch technology taking into account the findings of the original 2003 Venkatesh et al. study as a frame for data analysis.

The UTAUT was initially proposed by Venkatesh et al. who recognized a need to create a "unified view of user acceptance" (Venkatesh et al., 2003) in order to address the problem faced by researchers forced to " pick and choose' constructs across models... and largely ignore the contributions from alternative models" (Venkatesh et al., 2003). UTAUT is a combination of eight models that look at technology acceptance and takes a factor from every model to integrate in a unified model (Venkatesh et al., 2003). 
The theory covers:

1. Theory of Reasoned Action (TRA)

2. Technology Acceptance Model (TAM)

3. Motivational Model (MM)

4. Theory of Planned Behavior (TPB)

5. Combined TAM and TPB (C-TAM-TPB)

6. Model of PC Utilization (MPCU)

7. Innovation Diffusion Theory (IDT)

8. Social Cognitive Theory (SCT)

Taking into consideration specific areas in these theories and models helped to formulate a theory that covers most aspects of the user acceptance models (Venkatesh et al., 2003). Venkatesh and colleagues therefore propose the Unified Theory of Acceptance and Use of Technology as a model comprised of seven factors. These factors are summarized in the table below.

Table 1. Seven Factors of the Unified Theory of Acceptance and Use of Technology

\begin{tabular}{|c|c|}
\hline Factor & $\begin{array}{l}\text { Description } \\
\end{array}$ \\
\hline Performance Expectancy & $\begin{array}{l}\text { "The degree to which an individual believes that using } \\
\text { a system will help him or her to attain gains in job } \\
\text { performance" (Venkatesh et al., 2003, p. 447). } \\
\text { Performance Expectancy addresses user perception } \\
\text { towards a technology's ability to perform job tasks } \\
\text { with greater accuracy and efficiency than prior } \\
\text { technologies. }\end{array}$ \\
\hline Effort Expectancy & $\begin{array}{l}\text { "The degree of ease associated with the use of the } \\
\text { system" (Venkatesh et al., 2003). Effort expectancy } \\
\text { pertains to a user's perception of the ease or difficulty } \\
\text { of using a technology as compared to the prior } \\
\text { technology. }\end{array}$ \\
\hline Social Influence & $\begin{array}{l}\text { "The degree to which an individual perceives that } \\
\text { important others believe he or she should use the new } \\
\text { system" (Venkatesh et al., 2003). Critical to the } \\
\text { understanding of social influence are three } \\
\text { mechanisms: compliance, internalization, and } \\
\text { identification (Venkatesh et al., 2003). (This factor will } \\
\text { be further discussed below.) }\end{array}$ \\
\hline Facilitating Conditions & $\begin{array}{l}\text { "The degree to which an individual believes that an } \\
\text { organizational and technical infrastructure exists to } \\
\text { support use of the system" (Venkatesh et al., 2003). } \\
\text { Facilitating conditions include such factors as } \\
\text { organizational support for a technology or proximity of } \\
\text { technical support. }\end{array}$ \\
\hline Attitude Toward Using Technology & $\begin{array}{l}\text { "An individual's overall affective reaction to using a } \\
\text { system" (Venkatesh et al., 2003). This factor includes } \\
\text { elements such as a person's feelings about a } \\
\text { technology and the technology's relationship with the } \\
\text { user's attitude toward the task for which the technology } \\
\text { is employed (Venkatesh et al., 2003). }\end{array}$ \\
\hline Self-efficacy & $\begin{array}{l}\text { Self-efficacy refers to a person's belief or confidence } \\
\text { in their ability to use the technology to complete a task } \\
\text { based upon both internal and external factors. } \\
\text { (Venkatesh et al., 2003) }\end{array}$ \\
\hline Anxiety & $\begin{array}{l}\text { A user's fear of the use of the technology. In particular } \\
\text { the user's fear of making a mistake through the use of }\end{array}$ \\
\hline
\end{tabular}


Using the UTAUT as a theoretical basis, the researchers developed a survey-based study to answer the following research question:

RQ 1: How is the act of habitually checking messages and alerts on a smartwatch perceived by others engaged in a face-to-face interaction with the smartwatch-wearer?

\section{RESEARCH METHODOLOGY}

\section{Sample and Recruitment of Participants}

The scope of this study is confined to undergraduate and graduate students in the Information Systems program of a small regional university located in the northeastern United States. A written copy of the survey was administered to students in on-ground classes. The inclusion of undergraduate and graduate students provides a broad range of age, gender, professional culture and ethnic cultural background. This is important in assessing the hypothesis that each of these factors contributes to a person's attitude towards smartwatch technology.

\section{Data Collection and Analysis}

As previously stated, this study used a written survey consisting of open-ended qualitative questions and quantitative multiple choice and yes/no questions. The survey uses questions designed to address the UTAUT-recognized areas of "Attitude" and "Social Influence" in relation to the perceptions of the use of smartwatch technology. While the focus for this study is undergraduate and graduate Information Systems students, the survey questions are designed for use with any population. The goal of this survey is to determine what factors contribute to the perceptions of smartwatch technology. The focus on "Attitude" and "Social Influence" factors for this study results from the interests of the researchers to understand how potential cultural factors (if any) impact the acceptance of smartwatch technology. Such factors could include age, country of origin, sex, or socio-economic background, as well as other factors. The authors of this study believe that contrary to the proposed notions of Venkatesh and colleagues, "Attitude" and "Social Influence" are key factors in technology use and acceptance. This study represents the first step of a broader study intended to cover participants of differing cultural backgrounds.

Both the quantitative and qualitative sections of the survey include questions geared towards the understanding of each participant's attitude toward and perception of the use of smartwatch technology. These questions are designed to assess the attitudes of both participants that own and use smartwatches and those that do not use them but are exposed to the technology. The study focuses on the interactions between people in which one or both are wearing smartwatches. By including the results of those participants that do not own smartwatches, it is hoped to capture the attitudes of smartwatch owners and non-owners for comparisons about how each group perceives the behaviors associated with smartwatch technology usage. Also included in the survey is a demographic section focused on determining the role that factors such as age, gender, profession and national identity play in understanding participant perceptions of smartwatch technology.

\section{RESULTS}

As previously stated, this study's participant body consisted of undergraduate and graduate students from a small university located in the northeastern United States. In total 176 students participated in the survey. However, for each survey question not all students marked an answer. This was particularly the case for participant age and place of origin. While this circumstance impacts the ability to use the survey results in a generalized analysis for the student community at this particular university, it does not impact the significance of the survey results as an indicator of social trends with smartwatch technologies. 
The demographic breakdown for the participants who recorded sex is summarized below in Table 2 .

Table 2. Participant Demographics by Gender

\begin{tabular}{|c|c|c|}
\hline Female & Male & Total Reporting Sex \\
\hline 25 & 136 & 161 \\
\hline \multicolumn{2}{|c|}{ Total Participants in Study } & 176 \\
\hline
\end{tabular}

While the difference of participants reporting gender compared to the total number of participants varies by $9 \%$, the authors believed that a population of 161 participants who recorded gender in their surveys allowed for a comparison of results for the survey questions in order to determine if gender played a role in smartwatch technology acceptance and perceptions of usage. The results for survey questions 4 through 15 are presented below in conjunction with discussion of the individual question results.

\section{Participants' Rationale for Owning a Smartwatch}

The results for Question 4, as follow in Table 3, are of particular interest when linked to the participant answers to Question 5 as displayed in Table 4 below.

Table 3. Question 4 Results

\begin{tabular}{|r|c|c|c|}
\hline \multicolumn{1}{|l|}{ Do you have a smartwatch? } & \multicolumn{3}{l|}{} \\
\hline Gender & Yes & No & Total \\
\hline Male & 19 & 117 & 136 \\
\hline Female & 4 & 21 & 25 \\
\hline Total & 23 & 138 & 161 \\
\hline
\end{tabular}

Table 4. Question 5 Results

\begin{tabular}{|r|c|c|c|c|c|}
\hline How many of your peers own/use a smartwatch? & \multicolumn{1}{l|}{} \\
\hline Count & $\mathbf{0}$ & $\mathbf{1 - 5}$ & $\mathbf{5 - 1 0}$ & $\mathbf{1 0}+$ & Total \\
\hline Male & 43 & 79 & 7 & 5 & 134 \\
\hline Female & 7 & 17 & 1 & 0 & 25 \\
\hline Total & 50 & 96 & 8 & 5 & 159 \\
\hline
\end{tabular}

While the number of male students in the sampling is significantly higher than female students (at a ratio of slightly more than 5:1), the overall percentages of male and female students that own smartwatches versus not owning are consistent between both genders with 4 out of 5 male and female students not owning smartwatches. Furthermore, both male and female students reported by similar ratios of almost 2 to 1 that they had between 1 and 5 peers with smartwatches versus all other categories. Based on this student population sampled, there appears to be no gender difference in students owning smartwatch devices.

Survey questions 6 and 7 addressed the rationale for the purchase and use of smartwatches. Again, while the results of this study may not be generalizable, they do demonstrate that social trends and peer influence played little role in the participants' (both male and female) reasons for purchasing smartwatches. The inclusion of the results of those participants that do not own smartwatches are equally as valuable as they contribute to the understanding of the factors that would contribute to both smartwatch ownership as well as the participants' views of smartwatch usage during interactions with others. Of the listed reasons, functionality and communication were key factors. The "Other" category consisted of various individual reasons for using a smartwatch, demonstrating a level of individualism in the participant's usage for the smartwatch device. The complete results for survey questions 6 and 7 are presented in tables 5 and 6 below. 
Table 5. Question 6 Results

\begin{tabular}{|c|c|c|c|}
\hline Gender & Yes & No & Total \\
\hline Male & 3 & 123 & 126 \\
\hline Female & 1 & 20 & 21 \\
\hline Total & 4 & 143 & 147 \\
\hline
\end{tabular}

Table 6. Question 7 Results

\begin{tabular}{|l|c|c|r|}
\hline Why do you use a smartwatch? & \multicolumn{1}{c|}{} \\
\hline \multicolumn{1}{|c|}{ Gender } & Male & Female & Total \\
\hline Enjoy various functions and apps & 31 & 8 & 39 \\
\hline Convenient to communicate with others & 21 & 1 & 22 \\
\hline To get instant news and information & 18 & 4 & 22 \\
\hline Peer influence & 2 & 1 & 3 \\
\hline Follow the trend & 6 & 0 & 6 \\
\hline Other: (Please Elaborate) & 44 & 8 & 52 \\
\hline Total & 122 & 22 & 144 \\
\hline
\end{tabular}

\section{Participant Perceptions of Smartwatch Use in Social Situations}

When asked to evaluate perceptions of smartwatch use in social situations, the participants' responses demonstrate an interesting trend. As the results from survey questions 8 and 9 (presented below in tables 7 and 8) show, the majority of participants, both male and female, stated that when receiving notifications on their smartwatches while engaged in a face-to-face interaction with another person, they would not check the smartwatch notification as this would be considered rude to the other party in the conversation.

However, when the scenario is reversed and it is the other party in the face-to-face encounter that checks the smart phone (survey questions 11 and 12), the male survey participants stated almost 2:1 that they did not mind this behavior versus finding it rude. Furthermore 3:1 of the male participants never thought about the behavior compared to thinking it rude. The female survey participants, however, were almost evenly split in a 1:1:1 ratio of finding the behavior rude, not minding it, or not even thinking about it. Again, the small sampling population for this study means that this finding is not generalizable, but it is still significant and important for the intent of this study.

The full results for survey questions $8,9,11$, and 12 can be found in tables 7-10 below.

Table 7. Question 8 Results

If you receive a notification on your smartwatch while engaging in a face-to-face interaction with another person do you check your watch while interacting?

\begin{tabular}{|r|c|c|c|}
\hline Gender & Yes & No & Total \\
\hline Male & 31 & 75 & 106 \\
\hline Female & 5 & 13 & 18 \\
\hline Total & 36 & 88 & 124 \\
\hline
\end{tabular}

Table 8. Question 9 Results

\begin{tabular}{|l|c|c|c|}
\hline \multicolumn{1}{|c|}{ Phat is your perception of this behavior? } & Male & Female & Total \\
\hline \multicolumn{1}{|c|}{ Partipant Answer } & 52 & 10 & 62 \\
\hline You feel that you are acting rudely & 31 & 5 & 36 \\
\hline You never thought about it & 11 & 3 & 14 \\
\hline You don't think anyone minds & 7 & 1 & 8 \\
\hline It is normal behavior since everyone has a smartwatch & 4 & 0 & 4 \\
\hline You like that it draws attention to the fact that you have a smartwatch
\end{tabular}


Table 9. Question 11Results

Have you ever been in a face-to-face interaction with someone wearing a smartwatch and observed them checking notifications while interacting with you?

\begin{tabular}{|r|c|c|c|}
\hline Gender & Yes & No & Total \\
\hline Male & 54 & 67 & 121 \\
\hline Female & 13 & 11 & 24 \\
\hline Total & 67 & 78 & 145 \\
\hline
\end{tabular}

Table 10. Question 12 Results

\begin{tabular}{|c|c|c|c|}
\hline \multicolumn{4}{|l|}{ What is your perception of this behavior? } \\
\hline Participant Answer & Male & Female & Total \\
\hline You feel the person is acting rudely & 33 & 7 & 40 \\
\hline You never thought about it & 19 & 6 & 25 \\
\hline You don't mind & 58 & 9 & 67 \\
\hline It is normal behavior since everyone has a smartwatch & 4 & 0 & 4 \\
\hline You notice the smartwatch and wish you had one & 3 & 0 & 3 \\
\hline Total & 117 & 22 & 139 \\
\hline
\end{tabular}

\section{Integration of Smartwatch into Participants' Behavior Patterns}

Finally, this survey assessed how the participants integrated smartwatches into their daily behavioral patterns. Survey questions 10 and 13 addressed these patterns for both the smartwatch users and those interacting with the smartwatch user. In response to survey question 13, which asked the participants how long they wait to check a notification on their smartwatch, there was a split in ratios of responses between male and female participants. Female participants indicated a 1:1:1 ratio of checking the notification immediately, every 15 minutes, or every 30 minutes. Male survey participants, however, stated that they check their notifications immediately as compared to every 15 minutes by a ratio of $3: 1$. Those that indicated checking every 30 minutes compared to immediately were only $1: 10$.

Finally, $80 \%$ of both male and female participants indicated that other people seem to understand this notification checking behavior and are not confused by the action (survey question 10). The recorded participant results for survey questions 10 and 13 are represented in tables 11 and 12 below.

Table 11. Question 13 Results

\begin{tabular}{|l|c|c|c|}
\hline How often do you look at your smartwatch when a notification is received? & Male & Female & Total \\
\hline \multicolumn{1}{|c|}{ Participant Answer } & 38 & 3 & 41 \\
\hline Every time a notification goes off & 13 & 4 & 17 \\
\hline Every 15 minutes & 4 & 3 & 7 \\
\hline Every 30 minutes & 12 & 0 & 12 \\
\hline Every hour & 67 & 10 & 77 \\
\hline Total
\end{tabular}

Table 12. Question 10 Results

If you have checked your smartwatch during a face-to-face interaction, has the person you are interacting with ever appeared confused or uncomfortable with this action or commented on it?

\begin{tabular}{|r|c|c|c|}
\hline Gender & Yes & No & Total \\
\hline Male & 14 & 80 & 94 \\
\hline Tomale & 2 & 14 & 16 \\
\hline
\end{tabular}




\section{SUMMARY}

It is true that the number of participants that own smartwatches compared to the total population surveyed is small. This may be due to the cost of smartwatch devices relative to the income of the students surveyed. This is certainly an area for future study important to understand the economic barriers of smartwatch technology integration into different levels of society.

While it cannot be generalized beyond this study's population, within the context of the study population it appears that smartwatch behavior is becoming an accepted part of social interactions. The preponderance of literature in internet media proclaiming proper etiquette for smartwatch technology use further supports the findings of this study. The results indicating the self-conscious nature of smartwatch user conduct are of particular interest. Smartwatch users' belief that checking their watches while engaging others would be rude behavior yet accepting other smartwatch users' habits of checking their smartwatch device as an acceptable action requires further study. Understanding this conflicting pattern of human interaction is of particular relevance to understanding the acceptance of smartwatch technology into society. Again, the small sampling size for this study is not generalizable, but it can serve as a sampling for future studies. Factors such as age, differing national or regional cultures and professional cultures must be considered and compared.

Finally, the role of gender in the acceptance of smartwatch technology merits future study. Non-generalizable results aside, the results of this study indicated possible gender differences in assessing smartwatch use behaviors. Additionally, different gender attitudes in integration of smartwatch notification-checking conduct is possible. Given the notion of "glanceability" referenced by Giang et al. (2015), both of these sets of results warrant future study in larger populations.

\section{REFERENCES}

Chen, C., Shih, H. (2014). A Study of the Acceptance of Wearable Technology for Consumers - An Analytical Network Process Perspective. International Journal of the Analytic Hierarchy Process, 1-5.

Davis, F. D. (1989). Perceived usefulness, perceived ease of use, and user acceptance of information technology. MIS quarterly, 319-340.

Deng, X. N., \& Christodoulidou, N. (2015). Understanding User Values of Wearable Computing.

Gao, Y., Li, H., \& Luo, Y. (2015). An empirical study of wearable technology acceptance in healthcare. Industrial Management \& Data Systems, 115(9), 1704-1723.

Giang, W. C., Shanti, I., Chen, H. Y. W., Zhou, A., \& Donmez, B. (2015, September). Smartwatches vs. Smartphones: A preliminary report of driver behavior and perceived risk while responding to notifications. In Proceedings of the 7th International Conference on Automotive User Interfaces and Interactive Vehicular Applications, pp. 154-161. ACM.

Guo, Y., Barnes, S., \& Le-Nguyen, K. (2015). Consumer Acceptance IT Products: An Integrative Expectationconfirmation Model.

Hofstede, G., Hofstede, G. J., \& Minkov, M. (2010). Cultures and organizations-software of the mind: intercultural cooperation and its importance for survival (3rd ed.): McGraw-Hill New York, NY. 
Hu, P. J., Chau, P. Y., Sheng, O. R. L., \& Tam, K. Y. (1999). Examining the technology acceptance model using physician acceptance of telemedicine technology. Journal of Management Information Systems, 91-112.

Kenney, B. (2015). 'Smart' Device, Smartwatch and Wearable Etiquette is Evolving. Retrieved from http://smartwatches.org/learn/smart-device-smartwatch-wearable-etiquette-evolving/

Kim, K. J., \& Shin, D. H. (2015). An acceptance model for smart watches: implications for the adoption of future wearable technology. Internet Research, 25(4), 527-541.

Kim, S. H. (2014). A Study on Adoption Factors of Korean Smartphone Users: A Focus on TAM (Technology Acceptance Model) and UTAUT (Unified Theory of Acceptance and Use of Technology).

Kim, Y., Kim, D., \& Wachter, K. (2012). Smartphones: User Engagement Motivations Effect on Their Value, Satisfaction, and Future Engagement Intention.

Lane, W., \& Manner, C. (2011). The impact of personality traits on smartphone ownership and use. International Journal of Business and Social Science, 2(17), 22-28.

Mekić, E., \& Özlen, M. K. (2014). Acceptance of Smartphones by Users in BiH through Extended Technology Acceptance Model. И ССЛЕД, 136.

Pan, D., Chen, N., \& Rau, P. L. P. (2013). The Acceptance and Adoption of Smartphone Use among Chinese College Students. In Cross-Cultural Design. Methods, Practice, and Case Studies (pp. 450-458). Springer Berlin Heidelberg.

Park, JJ, Rajwanshi, V., et al. (2014). Smartwatch Market: Consumers are smarter than smartwatches; Opportunities vs Barriers. J.P. Morgan: Asia Pacific Equity Research.

Park, S. Y. (2009). An analysis of the technology acceptance model in understanding university students' behavioral intention to use e-learning. Journal of Educational Technology \& Society, 12(3), 150-162.

Park, Y., \& Chen, J. V. (2007). Acceptance and adoption of the innovative use of smartphone. Industrial Management \& Data Systems, 107(9), 1349-1365.

Pearson, J., Robinson, S., \& Jones, M. (2014). It's About Time: Smartwatches as Public Displays.

Petrow, S. (2015). Digital Life: Is there Apple Watch etiquette? Retrieved from http://www.usatoday.com/story/tech/personal/2015/04/24/apple-watch-etiquette/26239551/

Spicer, A. (2015). Apple Watch: Smartwatches could make us even more self-obsessed. Retrieved from http://www.cnn.com/2015/03/10/opinions/apple-watch-self-obsessed/

Venkatesh, V., Morris, M. G., Davis, G. B., \& Davis, F. D. (2003). User acceptance of information technology: Toward a unified view. MIS Quarterly, 425-478.

Yang, H., Yu, J., Zo, H., \& Choi, M. (2016). User acceptance of wearable devices: An extended perspective of perceived value. Telematics and Informatics, 33(2), 256-269. 